British Journal of Psychiatry (1985), 146, 99-104

\title{
Correspondence
}

Correspondents should note that space is limited and shorter letters have a greater chance of publication. The Editors reserve the right to cut letters and also to eliminate multitudinous references. Please try to be concise. strictly relevant and interesting to the reader, and check the accuracy of all references in Journal style.

\section{DEPRESSION AND AFFECT-LADEN WORDS}

DEAR SiR,

Dr Firestone (Journal, October 1984, 145, 447) points out a potential flaw in our experimental procedure whereby we found differences between depressed and control subjects in the influence the hedonic tone of material brought to bear on the accuracy of its subsequent recognition (Journal. April 1984, 144, 376-382). He suggests, in effect, that the "intensity of affect" attaching to the unpleasant material was greater than that of the pleasant material, and that this explained its readier recognition.

We fully accept that the intensity of affect was greater in the unpleasant material. If the original sematic differential groupings are considered (Broadbent D. E. and Gregory M., 1967, Nature $215,581-584)$ then the following values are seen:bad words - class boundaries 3.09-1.00, mean score 1.54; good words - class boundaries 7.00-4.80, mean score 5.89

Thus, the unpleasant material was associated with more intense affect than the pleasant material, but only to a small degree.

However, we do not accept that this explains our findings. The depressed subjects established stronger memory traces for the unpleasant material whereas the non-depressed controls showed precisely the reverse (Table $\mathrm{V}$ of our paper). If, as Dr Firestone postulates, intensity of affect is the sole explanation, one would then be in the curious situation that intensity of affects operates in the expected direction for depressives, but in the reverse direction for healthy controls. This would surely be unlikely.

A more parsimonious explanation is made in our paper. Thus, recognition performance is dependent on both the type/intensity of affect associated with the material and the mood state of the subject. If a congruence exists between the two, recognition is enhanced, when compared with a situation where this congruence is lacking.

\author{
W. A. Lishman \\ G. C. Dunbar
}

Institute of Psychiatry,

London SE5

THE USE OF LITHIUM IN SEVERELY DEMENTED PATIENTS WITH BEHAVIOURAL DISTURBANCE

DEAR SIR,

The problem of aggressive, overactive and agitated behaviour in the severely demented is common and difficult to manage. The usual approach is that of chemotherapy with neuroleptic medication which is not ideal for these behaviour problems. They have undesirable side-effects, especially in the elderly who, when brain damaged, are also prone to tardive dyskinesia.

Lithium has been used in mentally retarded patients with hyperactive and aggressive behaviour, (Souver \& Hurley, 1981; Goetzl et al, 1977). While the response may be because the behaviour stems from accompanying affective illness, it may be that lithium has a specific anti-aggressive effect (Sheard et al, 1976). In the elderly, lithium has been reported to be dramatically and rapidly effective in eight out of ten patients with organic brain syndrome, none of whom had been diagnosed as manic depressive (William \& Goldstein 1979). We carried out a pilot study on the feasibility of using low dosage lithium in patients suffering from severe chronic brain syndrome with aggressive, overactive behaviour or agitation normally requiring a neuroleptic to control.

Ten patients were selected, with an age range of $72-85$ years. Each patient had severe dementia with difficult behaviour. Seven patients completed the study. The other three became physically unwell during the trial and the lithium was therefore discontinued, although in none was lithium thought to be responsible. Neuroleptic medication was 